\title{
Surprises with the lattice index theorem
}

\author{
Gerald Jordan $^{a}{ }^{b}$, Manfried Faber ${ }^{a}$, Urs M. Heller ${ }^{c}$ and Roman Höllwieser ${ }^{a *}$ \\ ${ }^{a}$ Atomic Institute, Vienna University of Technology \\ Wiedner Hauptstr. 8-10, 1040 Vienna, Austria \\ ${ }^{b}$ Photonics Institute, Vienna University of Technology, Gusshausstrasse 25-29, 1040 Vienna, \\ Austrid American Physical Society, One Research Road \\ Box 9000, Ridge, NY 11961-9000, USA \\ E-mail: gerald@kph.tuwien.ac.at, faber@kph.tuwien.ac.at, \\ heller@aps.org, hroman@kph.tuwien.ac.at
}

The lattice index theorem for thick classical centre vortices with traces of plaquettes deviating at most by $1.5 \%$ from trivial plaquettes is investigated. For non-orientable spherical vortices, the index of the overlap Dirac operator differs from the topological charge defined by a lattice version of $F \tilde{F}$. The reason is the singular nature of the corresponding continuum gauge field.

The XXV International Symposium on Lattice Field Theory

July 30-4 August 2007

Regensburg, Germany

${ }^{*}$ Speaker. 


\section{Introduction}

The centre vortex model [1] has been proposed as an explanation of confinement in nonabelian gauge theories. Centre vortices, quantised magnetic flux lines, compress the gluonic flux into tubes and cause a linearly rising potential at large separations. Numerical evidence has been produced to support this assumption [2], and in addition, simulations have indicated that vortices could also account for phenomena related to chiral symmetry, such as topological charge and spontaneous chiral symmetry breaking (SCSB) $[3,4,5,6]$.

These non-perturbative features of the QCD vacuum are intimately linked to the properties of the low-lying spectrum of the Dirac operator. The Atiyah-Singer index theorem [7] states that the topological charge of a gauge field equals the index of the Dirac operator, while the Banks-Casher relation [8] sets the spectral density of the near-zero modes proportional to the chiral condensate, the order parameter for SCSB.

The fundamental problems of investigating chiral symmetry on the lattice have been overcome by the invention of overlap fermions. The overlap operator obeys the Ginsparg-Wilson relation and features an exact chiral symmetry [5]. It further implements a lattice version of the index theorem [6], and may even be used for the definition of a local topological charge density [3].

In this paper, we work with $\mathrm{SU}(2)$ and report on our calculations with the overlap operator applied to thick classical centre vortices in the shapes of planes (closed by lattice periodicity) and spheres. We investigate the localisation of zero-modes with respect to the position of the thick vortices and find an interesting discrepancy in the topological charge determined by different methods. The details of the individual vortex types will be discussed along with our results.

\section{Topological Charge}

We compare different definitions of the lattice topological charge:

(1) The topological charge of the continuum gauge field configuration for our rather simple vortex geometries amounts to adding up the contributions from the intersection points, which according to the colour orientation carry a charge $Q= \pm \frac{1}{2}$.

(2) The index of the overlap Dirac operator [6,9]. According to the Atiyah-Singer index theorem the topological charge is given by the index

$$
\text { ind } D[A]=n_{-}-n_{+}=Q
$$

where $n_{-}$and $n_{+}$are the number of left- and right-handed zeromodes of the Dirac operator [7]. The overlap Dirac operator is defined by

$$
D=\frac{1}{2}\left[1+\gamma_{5} \varepsilon\left(H_{L}^{+}\right)\right]
$$

Here, $\varepsilon$ is the sign function, $H_{L}^{+}=\gamma_{5} D_{w}\left(-m_{0}\right)$ and $D_{w}$ is the usual lattice Wilson Dirac operator with mass $-m_{0}$ (we use $m_{0}=1.0$ ). It has been shown that this fermionic definition of $Q$ coincides with the continuum simple gluonic definition in the continuum limit [10]. 
The lattice version of the index theorem is only valid as long as the gauge field satisfies a socalled "admissibility" condition. This condition assures that $H_{L}^{+}$has no zero eigenvalues so that the sign-function is well-defined. It requires that the plaquette-values $U_{\mu \nu}$ are bounded close to trivial $\mathbb{1}$. A sufficient, but not necessary bound for the "admissibility" of the gauge field is $[11,12]$

$$
\operatorname{tr}\left(\mathbb{1}-U_{\mu v}\right)<0.03
$$

(3) The gluonic charge in the plaquette and hypercube definition [13], measured after cooling.

With the field strength tensors $\mathscr{F}_{\mu v}(x)=\partial_{\mu} \mathscr{A}_{v}-\partial_{v} \mathscr{A}_{\mu}+\mathrm{i}\left[\mathscr{A}_{\mu}, \mathscr{A}_{v}\right]$ and $\tilde{\mathscr{F}}_{\mu \nu}=\frac{1}{2} \varepsilon_{\mu \nu \rho \sigma} \mathscr{F}_{\rho \sigma}$ the quantity which determines the affiliation to a homotopy class, the topological charge $Q$ or (negative) Pontryagin index reads for trivial boundary conditions[14, 15]

$$
\begin{aligned}
Q & \equiv \int d^{4} x q(x)=-\frac{1}{16 \pi^{2}} \int d^{4} x \operatorname{tr}\left(\mathscr{F}_{\mu \nu} \tilde{\mathscr{F}}_{\mu v}\right)= \\
& =-\frac{1}{8 \pi^{2}} \varepsilon_{\mu \nu \rho \sigma} \int d^{4} x \operatorname{tr}\left\{\left[\partial_{\mu} \mathscr{A}_{v}+\mathrm{i} \mathscr{A}_{\mu} \mathscr{A}_{v}\right]\left[\partial_{\rho} \mathscr{A}_{\sigma}+\mathrm{i} \mathscr{A}_{\rho} \mathscr{A}_{\sigma}\right]\right\}= \\
& =-\frac{1}{8 \pi^{2}} \varepsilon_{\mu \nu \rho \sigma} \int d^{4} x \operatorname{tr}\left\{\partial_{\mu} \mathscr{A}_{v} \partial_{\rho} \mathscr{A}_{\sigma}+2 \mathrm{i} \mathscr{A}_{\mu} \mathscr{A}_{v} \partial_{\rho} \mathscr{A}_{\sigma}\right\} .
\end{aligned}
$$

The topological charge density $q(x)$ is the total derivative of the topological current $k_{\mu}$

$$
q(x)=\partial_{\mu} k_{\mu}, \quad k_{\mu}=-\frac{1}{8 \pi^{2}} \varepsilon_{\mu v \rho \sigma} \operatorname{tr}\left[\mathscr{A}_{v} \partial_{\rho} \mathscr{A}_{\sigma}+\mathrm{i} \frac{2}{3} \mathscr{A}_{v} \mathscr{A}_{\rho} \mathscr{A}_{\sigma}\right] .
$$

For smooth gauge fields one can apply the Gauss-theorem to transform the expression for $Q$ into a surface integral

$$
Q=\int d^{4} x q(x)=\oint_{S^{3}} d \sigma_{\mu} k_{\mu} .
$$

For $x^{2} \rightarrow \infty$ we assume a sufficiently fast decaying field strength, so that $\mathscr{F}_{\mu \nu}=0$ implies $\varepsilon_{\mu \nu \rho \sigma} \partial_{\rho} \mathscr{A}_{\sigma}=-\mathrm{i} \varepsilon_{\mu \nu \rho \sigma} \mathscr{A}_{\rho} \mathscr{A}_{\sigma}$. Thus we obtain

$$
Q=\frac{\mathrm{i} \varepsilon_{\mu v \rho \sigma}}{24 \pi^{2}} \oint_{S^{3}} d \sigma_{\mu} \operatorname{tr}\left[\mathscr{A}_{v} \mathscr{A}_{\rho} \mathscr{A}_{\sigma}\right]
$$

By a gauge transformation $\Omega(x)$ we can put the free gauge field at infinity to zero

$$
0=\mathscr{A}_{\mu}^{\prime}\left(x^{2} \rightarrow \infty\right)=\Omega^{\dagger}(x)\left[\mathscr{A}_{\mu}(x)-\mathrm{i} \partial_{\mu}\right] \Omega(x) \quad \Longleftrightarrow \quad \mathscr{A}_{\mu}\left(x^{2} \rightarrow \infty\right)=\mathrm{i} \partial_{\mu} \Omega \Omega^{\dagger} .
$$

The pure gauge potential $\mathscr{A}_{\mu}$ in Eq. (2.10) maps a 3D volume element $d x_{v} d x_{\rho} d_{\sigma}$ at infinity to a volume element

$$
\mathrm{i} \mathscr{A}_{v} \mathscr{A}_{\rho} \mathscr{A}_{\sigma} d x_{v} d x_{\rho} d_{\sigma}=\partial_{v} \Omega \Omega^{\dagger} \partial_{\rho} \Omega \Omega^{\dagger} \partial_{\sigma} \Omega \Omega^{\dagger} d x_{v} d x_{\rho} d_{\sigma}
$$

in $\mathrm{SU}(2)$ group space. The requirement that the gauge transformation $\Omega(x)$ is smooth implies that the topological charge $Q$ in Eq. (2.9) is integer, $Q \in \mathbb{Z}$. $Q$ measures the number of times $\Omega(x)$ wraps around the group when $x$ covers the spacetime surface $S^{3}$ at $x^{2} \rightarrow \infty$ once. Choosing bases in the tangent spaces of the two $S^{3}$ manifolds, we can define an orientation of the map. Thus $Q$ can take positive and negative values. 

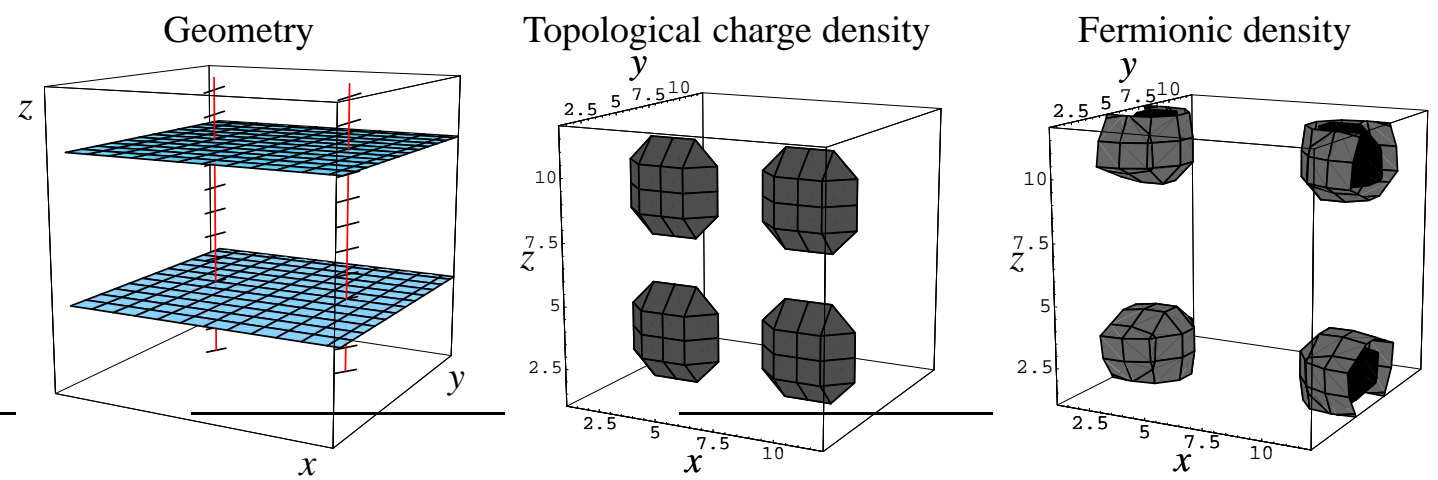

Figure 1: Plane vortices on a $12^{4}$-lattice in xy- and zt-planes intersect in four points giving rise to topological charge and localised fermionic density.

\section{Description of configurations and results}

\subsection{Plane vortices}

Planar vortices are constructed as explained in [16]. At periodic boundary conditions they always come in pairs and therefore are automatically closed. All three definitions of $Q$ yield identical results for all configurations containing only plane vortices.

In fig. 1 we show the topological charge density for a gauge field consisting of 2 orthogonal pairs of plane vortices, which intersect in 4 points, each of which gives rise to a lump of topological charge $Q=\frac{1}{2}$. We also show the scalar fermionic density of the two zeromodes. Our result is in agreement with the analytical solution for the zeromodes presented in [17].

\subsection{Spherical vortices}

We distinguish between an orientable and a non-orientable spherical vortex.

1. The non-orientable spherical vortex of radius $R$ and thickness $\Delta$ is constructed with the following links:

$$
\begin{gathered}
U_{\mu}\left(x^{v}\right)= \begin{cases}\exp \left(\mathrm{i} \alpha\left(\left|\vec{r}-\vec{r}_{0}\right|\right) \vec{n} \cdot \vec{\sigma}\right) & t=1, \mu=4 \\
\mathbb{1} & \text { elsewhere }\end{cases} \\
\vec{n}(\vec{r}, t)=\frac{\vec{r}-\vec{r}_{0}}{\left|\vec{r}-\vec{r}_{0}\right|}
\end{gathered}
$$

where the function $\alpha$ is either one from $\alpha_{+}, \alpha_{-}$, which are defined as

$$
\begin{aligned}
& \alpha_{+}(r)= \begin{cases}0 & r<R-\frac{\Delta}{2} \\
\frac{\pi}{2}\left(1-\frac{r-R}{\frac{\Delta}{2}}\right) & R-\frac{\Delta}{2}<r<R+\frac{\Delta}{2} \\
\pi & R+\frac{\Delta}{2}<r\end{cases} \\
& \alpha_{-}(r)= \begin{cases}\pi & r<R-\frac{\Delta}{2} \\
\frac{\pi}{2}\left(1+\frac{r-R}{\frac{\Delta}{2}}\right) & R-\frac{\Delta}{2}<r<R+\frac{\Delta}{2} \\
0 & R+\frac{\Delta}{2}<r\end{cases}
\end{aligned}
$$


a)

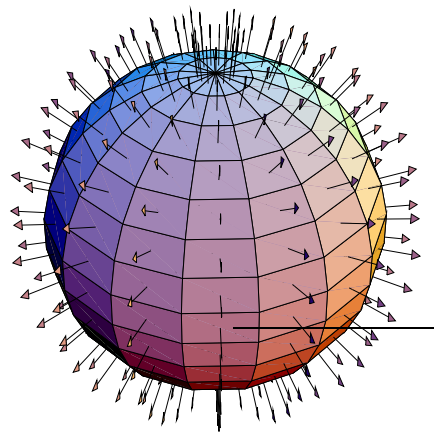

linkphase

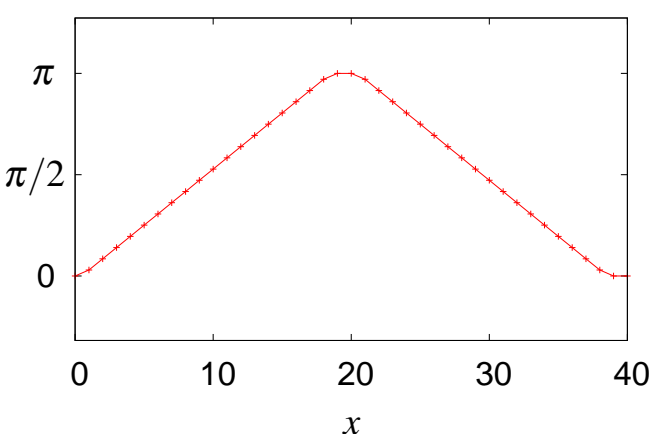

Figure 2: Thick spherical SU(2)-vortex (hedgehog, non-orientable) and variation of its link phase $\left(\alpha_{-}\right)$

This means that all links are equal to $\mathbb{1}$ except for the $t$-links in a single time-slice at fixed $t=1$. The phase changes from 0 to $\pi$ from inside to outside (or vice versa). The graph of $\alpha_{-}(r)$ for our largest lattice $40^{3} \times 2$ is shown in fig. $2 \mathrm{~b}$. The traces of all plaquettes are close to unity, $\operatorname{tr}\left(\mathbb{1}-U_{\mu v}\right) \leq 1-\cos \frac{\pi}{18}=0.015$. In our computations, $R$ is set to half the lattice size, and $\Delta$ is chosen such that only 3 links along any direction are equal to $+\mathbb{1}$ and $-\mathbb{1}$, respectively. The colour vector $\vec{n}$ changes according to the spatial direction (see fig. 2a).

2. The orientable vortex is constructed in a similar way:

$$
U_{\mu}\left(x^{v}\right)= \begin{cases}\exp \left(i \alpha\left(\left|\vec{r}-\vec{r}_{0}\right|\right)\left|n_{i}\right| \sigma_{i}\right) & t=1, \mu=4 \\ \mathbb{1} & \text { elsewhere }\end{cases}
$$

The distinction non-/orientable [18] refers to the orientation of the vortex surface assigned by abelian projection. While the orientable vortex has a global orientation, the non-orientable vortex consists of 2 patches of opposite orientation separated by a closed monopole worldline (fig. 3).
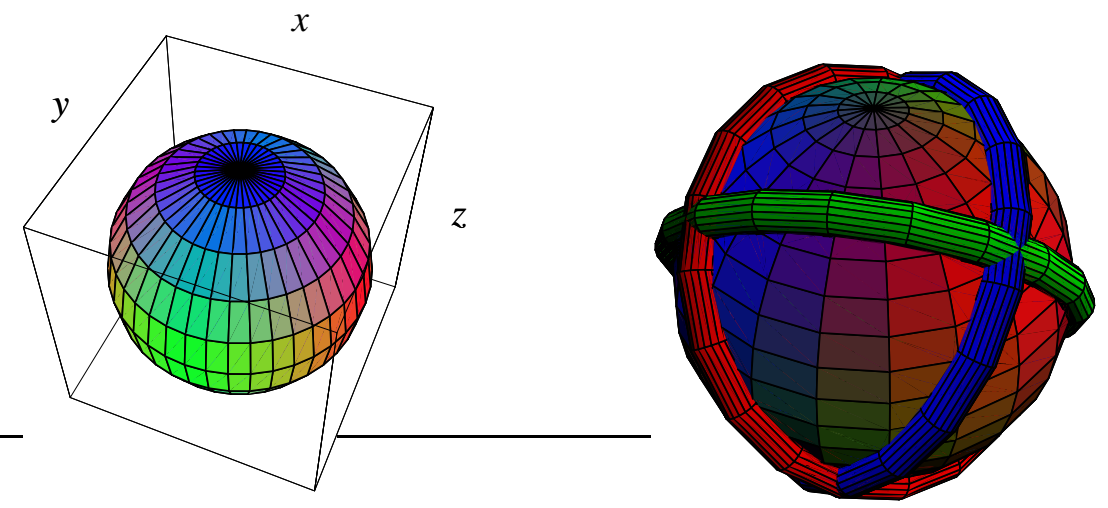

Figure 3: Non-orientable vortex surface (1) leads to monopole lines after abelian projection (r)

For a spherical vortex alone, the topological charge measured on the unsmoothed links is vanishing, since only the $U_{t \alpha}, \alpha=x, y, z$ plaquettes are non-zero, which gives a zero

$$
Q \sim \varepsilon_{\mu v \rho \sigma} U_{\mu \nu} U_{\rho \sigma}
$$


orientable vortex

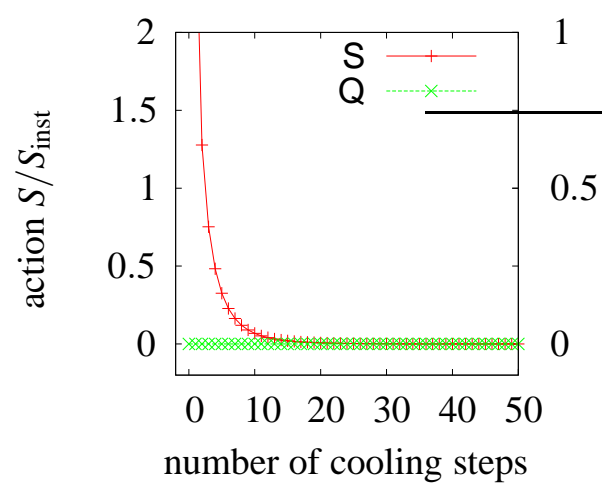

non-orientable vortex

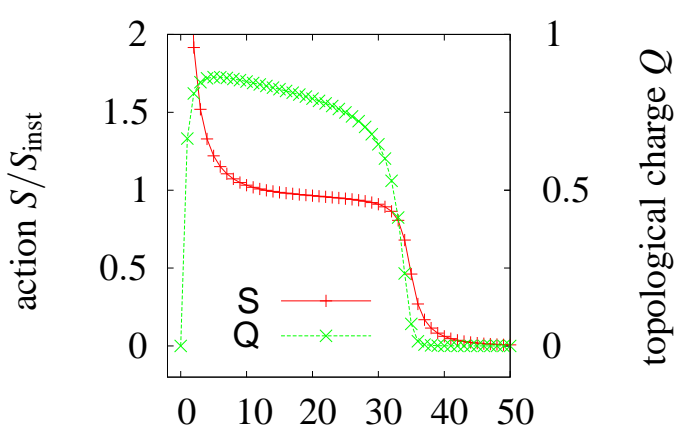

number of cooling steps

Figure 4: For configurations with an orientable vortex the action vanishes during cooling and no topological charge is measured (1), whereas for non-orientable vortex configurations the topological charge rises to 1 while the action $S$ reaches a (non-zero) plateau (r). The data is taken from a $12^{4}$-lattice.

This is independent of the lattice constant and thus holds also in the continuum limit.

For the orientable vortex, the topological charge after cooling and the overlap index are also equal to zero, in keeping with the continuum expectation (see left diagram of fig. 4).

However, we find a discrepancy in the case of the non-orientable sphere vortex.

First, during cooling the topological charge rises near to \pm 1 for $\alpha_{ \pm}$(right diagram of fig. 4 ) while the action $S$ reaches a (non-zero) plateau. Further, the index of the overlap operator is also non-zero, ind $D=\mp 1$ for $\alpha_{ \pm}$. Details are given in the table below:

\begin{tabular}{|l|r|r|r|}
\hline type & $n_{+}$ & $n_{-}$ & ind $D=n_{-}-n_{+}$ \\
\hline non-orientable, $\alpha_{-}$ & 3 & 4 & 1 \\
non-orientable, $\alpha_{+}$ & 1 & 0 & -1 \\
orientable, $\alpha_{ \pm}$ & 0 & 0 & 0 \\
\hline
\end{tabular}

The non-orientable vortex also gives extra contributions to the index when it is combined with other vortices, possibly including intersection points which produce "real" topological charge.

The discrepancy between overlap index and continuum topological charge is not due to the coarse discretisation. We have used lattice sizes with $N_{t}=2$ and $N_{s}$ ranging from 8 to 40 in steps of 4 [19]. For $N_{s} \geq 40$, we satisfy the admissibility condition (2.3), but our results remain unaltered.

More generally, the following empirical rule can be formulated: Non-orientable spherical vortices in slices (3D volumes) of the lattice contribute to cooled topological charge and Dirac operator index with an integer given by the "winding number" [9] of the corresponding Wilson lines, mapping the 3D volume of the slice to the SU(2) manifold of the Wilson lines. To compute this "winding number", the $t$-links are seen as a map not from $\mathbb{T}^{4}$, but from the compactified time-slice $t=0$, in which the sphere is located, to $\mathrm{SU}(2)$. The time-slice can be compactified to $S^{3}$ because the links outside the sphere are all equal to $+\mathbb{1}$. 


\section{Conclusion}

For non-orientable spherical vortices, the index of the overlap Dirac operator differs from the topological charge in the continuum limit. The reason for the seeming contradiction is the singular nature of the continuum gauge field equivalent to our spherical vortex. This singularity invalidates the usual derivation of the index theorem.

\section{References}

[1] G. 't Hooft, Nucl. Phys. B138, 1 (1978)

P. Vinciarelli, Phys. Lett. 78B, 485 (1978)

T. Yoneya, Nucl. Phys. B144, 195 (1978)

M. Cornwall, Nucl. Phys. B157, 392 (1979)

G. Mack, V. B. Petkova, Ann. Phys. (NY) 123, 442 (1979)

B. Nielsen, P. Olesen, Nucl. Phys. B160, 380 (1979)

[2] L. Del Debbio, M. Faber, J. Greensite, S. Olejník, Phys. Rev. D55, 2298 (1997)

T. G. Kov'acs, E. T. Tomboulis, Phys. Rev. D57, 4054 (1998)

[3] M. Engelhardt, H. Reinhardt, arXiv:hep-th/0010031 (2000)

[4] R. G. Edwards, U. M. Heller, R. Narayanan, Phys. Rev. D59, 094510 (1999)

P. de Forcrand, M. D'Elia, Phys. Rev. Lett. 82, 4582 (1999)

C. Alexandrou, P. de Forcrand, M. D’Elia, Nucl. Phys. A663, 1031 (2000)

M. Engelhardt, Nucl. Phys. B638, 81 (2002)

[5] M. Lüscher, Phys. Lett. B428, 342 (1998)

[6] R. Narayanan, H. Neuberger, Nucl. Phys. B443, 305 (1995)

[7] M. Atiyah and I. M. Singer, Ann. Math. 93 (1971) 139; A. S. Schwarz, Phys. Lett. 67B (1977) 172;

L. Brown, R. Carlitz and C. Lee, Phys. Rev. D16 (1977) 417

[8] T. Banks, A. Casher, Nucl. Phys. B169, 103 (1980)

[9] H. Neuberger, Phys. Lett. B417, 141 (1998)

H. Neuberger, Phys. Lett. B427, 353, (1998)

[10] D. H.Adams, J. Math. Phys. 42, 12, 5522 (2001)

[11] M. Lüscher, Nucl. Phys. B549, 295 (1999);

[12] H. Neuberger, Phys. Rev. D61, 085015 (2000)

H. Fukaya, S. Hashimoto, T. Hirohashi, H. Matsufuru, K. Ogawa and T. Onogi, PoS LAT2005, 123 (2006) [arXiv:hep-lat/0510095].

[13] P. Di Vecchia et al., Nucl. Phys. B192, 392 (1981),Phys. Lett. B108, 323 (1982)

[14] Pierre van Ball, Commun. Math. Phys. 85 (1982) 529.

[15] H. Reinhardt, Nucl. Phys. B 503 (1997) 505 [arXiv:hep-th/9702049].

[16] M. Faber, J. Greensite, S. Olejník, JHEP 11, 53 (2001)

[17] H. Reinhardt, O. Schroeder, T. Tok, V. C. Zhukovsky, Phys. Rev. D66 085004 (2002)

[18] R. Bertle, M. Faber, J. Greensite and S. Olejník, JHEP 9903 (1999) 019 [arXiv:hep-lat/9903023].

[19] G. Jordan, R. Höllwieser, M. Faber and Urs M. Heller, Phys. Ref. D77 014515 (2008) 